Año: 2020

Fecha de aceptado: 29/8/2019

Correo: revista.societas@up.ac.pa

Numero: 1 Número de Páginas: 1-16

URL:https://revistas.up.ac.pa/index.php/societas

\title{
Influencia de la Evaluación Formativa en el Aprendizaje de los Idiomas Extranjeros
}

\author{
Influence of formative assessment on foreign language learning
}

\author{
María Célica Vivian Chamorro Guerrero.
}

Universidad Metropolitana de Educación Ciencia y Tecnología. Facultad de Educación. Doctorado en Ciencias de la Educación, Panamá, Panamá. Correo: macevister@gmail.com, https://orcid.org/0000-0002-2074-5670;

\begin{abstract}
Resumen
La enseñanza de los idiomas es un proceso que ha tomado diversos enfoques a través del tiempo en la educación; sin embargo, la evaluación se ha mantenido rezagada puesto que todavía se caracteriza por ser tradicional, estandarizada, homogénea y sumativa enfocada en el resultado mas no en el proceso; no obstante, este resultado no da cuenta integral del proceso de aprendizaje. En este sentido, este artículo aborda la evaluación formativa realizando una revisión referente a la evaluación de los idiomas extranjeros, describiendo su evolución y como factores como la ansiedad, la diversidad y la motivación inciden en su desarrollo y aplicación. Se profundiza en la evaluación formativa como practica para el aprendizaje analizando su respuesta a los factores que la afectan al igual que, como fomenta la autonomía, la autorregulación, la autoeficacia, la reflexión y el pensamiento crítico convirtiéndola en una evaluación humanizada y democrática.
\end{abstract}

Palabras clave: Evaluación; Evaluación Formativa; Autonomía; Retroalimentación.

\section{Abstract}

Foreign Language Teaching is a process that has taken different approaches over time in education; however, the evaluation has lagged since it is still characterized by being traditional, standardized, homogeneous and summative. It focuses on the result but not on the process; however, this result does not fully account for the learning process. In this sense, this article addresses formative assessment by conducting a review regarding the evaluation of foreign languages, describing its evolution and how factors such as anxiety, diversity and motivation affect its development and application. In a deep analysis, formative assessment is evaluation for learning. it is analyzed how formative assessment tackles the factors that affect it, as well as how it fosters autonomy, self-regulation, selfefficacy, reflection and critical thinking, turning it into a humanized and democratic evaluation. 
Keywords: Assessment; Formative Evaluation; Autonomy; Feedback.

\section{Introducción}

La enseñanza de los idiomas extranjeros es un proceso que se ve permeado por diferentes enfoques que la han direccionado a través del tiempo. Sin embargo, los procesos evaluativos no han ido a la par con este cambio, limitando su articulación con las prácticas de enseñanza aprendizaje, para ir más allá de la practica tradicional sumativa de medir el resultado y adentrarse en el proceso. En este sentido, el propósito de esta revisión es proveer un conocimiento detallado de la evaluación formativa en relación a los idiomas extranjeros, empezando por describir cómo se han llevado a cabo los procesos evaluativos, los factores que intervienen y los tipos de evaluación formativa. Asimismo, se discutirá sobre su influencia y amplitud para cubrir diversas dinámicas que afectan la enseñanza y evaluación de los idiomas extranjeros, y cómo su incidencia fortalece y potencia la motivación, la auto regulación y la autoeficacia fomentando la crítica, la reflexión y la democratización de la evaluación.

\section{Evaluación de los idiomas extranjeros}

La evaluación de los idiomas se refiere al proceso sistematico de evaluación y medición de los datos y la información recolectada a traves de pruebas orales y escritas acerca del conocimiento que los estudiantes tienen sobre el idioma, la compresion, y la habilidad, con el fin de mejorar su aprendizaje y desarrollo del lenguaje (Coombe, 2008) con el proposito de utilizarlo en contextos comunicativos reales. Es conveniente aclarar que, en la enseñanza de los idiomas, el termino evaluación se toma desde dos perspectivas: 'assessment' y 'evaluation. Sin embargo, estos términos en el español se toman como uno solo y con el mismo significado. De acuerdo al Ministerio de Educación Nacional (MEN, 1998), esta interpretación ha dificultado la comprensión de la dinámica del proceso y de los propósitos de una evaluación formativa. Las dos perspectivas antes mencionadas se definen así:

Assessment (Valoración): Proceso de observación y acumulación objetiva de evidencias del progreso individual de aprendizaje en los estudiantes.

Evaluation (Evaluación): Proceso en el cual se hacen estimaciones acerca de la pertinencia y efectividad de la enseñanza para el aprendizaje, sobre las bases de una valoración objetiva y veraz. La evaluación, en este contexto, es tanto un componente del proceso como el proceso mismo. (1998, p.21)

Ahora bien, también se debe tener claridad entre lo que es evaluar y lo que es examinar, ya que tienden a ser términos que se utilizan indistintamente para denotar un mismo proceso. Sin embargo, evaluar involucra la recogida de información tanto cuantitativa como cualitativa a nivel de pruebas y observaciones, clasificaciones según opinión y juicio de valor respectivamente. En cambio, examinar implica el procedimiento para medir las habilidades, el conocimiento o la actuación a través de exámenes que son útiles pero que no son las únicas herramientas que los maestros pueden usar para evaluar a los estudiantes. En consecuencia, el evaluar es mucho más amplio y complejo 
que examinar (Brown, 2004; Pastor, 2003). Se podría afirmar, que la incorrecta interpretación se debe a que por mucho tiempo la evaluación en general, se ha limitado a la aplicación del examen tradicional de cuestionario.

\section{La ansiedad hacia el idioma extranjero}

La ansiedad hacia el idioma extranjero se define como el sentimiento subjetivo de tensión, aprensión, nerviosismo y preocupación. Esta es considerada uno de los factores afectivos más importantes que influyen en el éxito del aprendizaje de idiomas (Horwitz, 2001). Este factor consiste en las autopercepciones, creencias, sentimientos y comportamientos en el aula que surgen de la singularidad del proceso de aprendizaje de idiomas (Horwitz, Horwitz y Cope, 1986). Cuando una situación requiere el uso del idioma extranjero, la ansiedad podría evidenciarse como el recelo del estudiante para usar el idioma, el miedo ante los exámenes y el miedo a una evaluación negativa por parte de otros individuos. De las cuatro habilidades lingüísticas escuchar, hablar, leer y escribir; el "hablar es el aspecto que más provoca ansiedad en una situación de aprendizaje de un segundo idioma" (Cheng, Horwitz y Schallert 1999, p. 40). En consecuencia, cuando un estudiante se enfrenta a los procesos de evaluación que por lo general son estandarizadas (tipo cuestionario y entrevista) resulta ser un proceso demasiado estresante para los estudiantes. De acuerdo con Horwitz, Horwitz y Cope (1986)

Los estudiantes que están ansiosos por los exámenes en la clase de idioma extranjero probablemente experimenten dificultades considerables ya que los exámenes y cuestionarios son frecuentes e incluso los estudiantes más brillantes y más preparados a menudo cometen errores. Las pruebas orales tienen el potencial de provocar ansiedad al examen y a la comunicación oral simultáneamente en estudiantes susceptibles. (p.128)

Katalin (2006) afirma que hay una especie de presión sobre los estudiantes por lo que todos parecen nerviosos, o, a pesar de haber estudiado, están estresados y, cuando se les hace una pregunta y se les pide que la respondan, es como si escucharan su sentencia de muerte. Por otro lado, esta situación puede afectar también la evaluación que realice el docente sobre el estudiante pues podría considerar que carece de alguna aptitud necesaria para aprender un idioma o motivación suficiente para desarrollar las actividades.

\section{Atención a la diversidad}

La diversidad es un concepto mucho más amplio que abarca no solo a los estudiantes con discapacidades sino a todos los estudiantes que forman parte del sistema educativo. Es decir, se toman en cuenta otros factores como origen cultural o social, la lengua, o incluso los propios estilos de aprendizaje (Paz, 2018; Torello y Olmos, 2012). La diversidad es una manera de pensar, de hacer, de actuar y de comprender la realidad (Torello y Olmos, 2012). En este sentido, "la diversidad se fundamenta en tomar en consideración las diferencias evidentes, no palpables, y las semejanzas entre los sujetos. Además, esta diversidad trata de la desemejanza dentro de una sociedad común 
y engloba a todos los individuos sin importar su condición" (Paz, 2018, p.69). En este orden de ideas, el docente debe mirar a la diversidad y en su atención a la inclusión términos que en la praxis son complementarios- no como un problema sino más bien como una oportunidad, un reto para enriquecer las formas de enseñar, de aprender y de evaluar (Herrera, Pérez y Echeita, 2016; Leiva, 2013). En este particular, se necesita moverse de contextos marcados por evaluaciones sumativas, tradicionales, estandarizadas hacia una evaluación que pueda ajustarse a las condiciones del estudiante y no viceversa. Por lo general, la evaluación involucra apuestas muy altas, ya que son determinantes para la aprobación de cursos, admisiones para la universidad, y para los procedimientos de reclutamiento laboral (Kormos, 2013). En consecuencia, se debe buscar garantizar la equidad en la evaluación de la competencia en el segundo idioma de los estudiantes con diferencias de aprendizaje específicas y discapacidades físicas.

En adición a esto, la evaluación va intrínsecamente ligada al proceso de enseñanza aprendizaje por lo que cuanto más tengan conocimiento los profesores sobre los estilos de aprendizaje de sus estudiantes, más eficazmente podrán orientar la enseñanza de la lengua extranjera, así como enlazar esos estilos a estrategias de enseñanza del idioma y diseñar los procesos evaluativos con base en esta información. Así, mientras que unos estudiantes puedan necesitar instrucción presentada más visualmente, otros pueden requerir instrucción auditiva, kinestésica o táctil. Sin un conocimiento adecuado sobre los estilos de aprendizaje individuales de aprendizaje, los maestros no pueden proporcionar sistemáticamente la variedad de instrucción adecuada (Oxford, 2003).

\section{Motivación}

La motivación juega un rol importante en el aprendizaje de los idiomas y por ende en su evaluación. La motivación se considera como el esfuerzo que los estudiantes ponen para aprender un idioma extranjero como resultado de su deseo o necesidad de aprenderlo (Ellis,1997; Saville-Troike y Barto, 2016). El estudiante puede aprender el idioma por aumentar las oportunidades ocupacionales, mejorar el prestigio y el poder, acceder a información científica y técnica o aprobar un curso en la universidad, el interés se genera por una actividad de aprendizaje externa en particular para recibir una recompensa externa, de otra persona (motivación instrumental o extrínseca); puede aprenderlo por el deseo de participar, integrarse e identificarse con la cultural del idioma (motivación integrativa); o puede aprenderlo por sus propias necesidades y objetivos auto percibidos (motivación intrínseca) (Brown, 2000; Ellis, 1997; Saville-Troike y Barto, 2016). En este orden de ideas, los procesos de enseñanza y evaluación convencionales (tradicionales y estandarizados) y los resultados que se obtienen de ellos podrían influir en la motivación (Rodriguez, 2012). Esta podría ser una de las causas de que el aprendizaje de los idiomas se ha convertido en un proceso difícil, estresante, e incluso agobiante. A nivel de educación terciaria, se aprende el idioma porque hace parte de los contenidos programáticos de una carrera profesional. En consecuencia, en muchos casos se aprende el idioma porque se necesita para aprobar y culminar los estudios 
superiores y no porque haya una motivación verdadera (Rodriguez, 2012; Larrenua, 2015).

\section{La evaluación formativa en la enseñanza y aprendizaje de los idiomas extranjeros}

La evaluación formativa también conocida como evaluación para el aprendizaje (Brown, 2004; Leung \& Lewkowicz, 2017; Mohan, 2012) ofrece un contexto diferente de evaluación debido a que permite recoger datos completos, no solo del resultado sino también del proceso, con el fin de obtener un panorama completo de la competencia comunicativa que va desarrollando el estudiante, en aras de un desarrollo cognitivo integral y de igual forma, que este proceso influya en su desarrollo personal. Así, "la evaluación formativa es diseñada para guiar de cerca el aprendizaje de los estudiantes" (Bailey, 2017, p.285). "La condición ideal es cuando todas las tareas de evaluación en el aula son formativas y para el aprendizaje. Como la evaluación se integra con el aprendizaje, la mayoría de las prácticas en el aula son formativas" (Clark, 2012, p.438). La característica principal de la evaluación formativa es "que la evidencia se evoca, se interpreta en términos de necesidades de aprendizaje y se usa para hacer ajustes para satisfacer mejor esas necesidades de aprendizaje" (Bailey, 2017, p. 285).

Asimismo, los maestros pueden hacer cambios pedagógicos y didácticos apropiados a mitad de curso para alcanzar objetivos de manera más efectiva gracias a que las evaluaciones son continuas y monitorean el desarrollo identificando las fortalezas y debilidades de todos los aspectos de la enseñanza y el aprendizaje de igual forma, la información recolectada puede ser utilizada como base para la planificación y acción futura con el fin de fortalecer y mejorar el plan de estudios (Breen y Candlin, 2001; Brown, 2002; Davis, Norris, Malone, y McKay, 2018; Mohan, 2012; Richards \& Renandya, 2002). Además, la evaluación formativa o continua, en lugar de basarse en algunos criterios prescritos como lo hace la evaluación sumativa puede dar forma, guiar el aprendizaje y guiar las decisiones dentro del proceso curricular con el fin de mejorar la enseñanza. Es decir,

cualquier evaluación compartida y negociada dentro del aula generará comentarios potencialmente formativos para y entre los alumnos y, entre los estudiantes y el profesor. La evaluación formativa puede no solo indicar los éxitos y fracasos relativos tanto del alumno como del plan de estudios, sino que también puede indicar direcciones nuevas y diferentes en las que ambos pueden moverse y desarrollarse (Breen y Candlin, 2001, p.23).

Además, como lo argumenta Bailey (2017), la evaluación formativa puede ser llevada a cabo por maestros de manera informal durante la clase como parte de la instrucción continua, o puede ser formal, es decir, planificada de antemano para abordar ciertos aspectos del conocimiento del idioma del estudiante. Por ejemplo, el uso de observaciones que pueden ser informales o pueden llegar a ser formales.

El uso de observaciones informales, por ejemplo, permite una variedad de habilidades (por ejemplo, el discurso oral entre pares) que no siempre son susceptibles de entornos de evaluación más formales o estandarizados. Las observaciones también se pueden hacer formalmente y utilizar para evaluar la 
calidad del entorno lingüístico de un aula en lugar de estudiantes individuales. (Bailey, 2017, p.329)

Adicionalmente, la evaluación formativa puede verse reflejada en el ambiente de aula, participación de los padres, los hábitos de alfabetización en el hogar, entre otros; al igual que puede tomar diversas formas: evaluación para el aprendizaje, evaluación holística, evaluación integrativa, entre otras (Bailey, 2017; Shohamy, 2017). Así dichas las cosas, la evaluación formativa en revisión profunda puede ser formal o informal y se compone de elementos integrativos, alternativos y auténticos para guiar de cerca el aprendizaje de los estudiantes (Bailey, 2017).

Además, por años, las evaluaciones se han desarrollado de manera tradicional con cuestionarios o pruebas estandarizadas fáciles de administrar, fáciles de calificar y fáciles de interpretar. Sin embargo, los resultados que se obtienen no logran dar un panorama amplio a los maestros para tomar decisiones sobre las necesidades de enseñanza o el progreso de sus estudiantes. En este sentido, la evaluación formativa al ocuparse del proceso brinda dentro de su repertorio técnicas alternativas que podrían dar respuesta a los diferentes aspectos cognitivos, afectivos, sociales y culturales que influyen en el aprendizaje tales como: estudiantes con necesidades especiales de aprendizaje, estilos de aprendizaje, diversidad, motivación, ansiedad, entre otros.

De acuerdo a Litchfield y Dempsey (2013), las técnicas alternativas de evaluación ofrecen tareas para desarrollar en lugar de que se limiten solo a contestar o seleccionar respuestas de un cuestionario.

Los estudiantes participan desarrollando las tareas que se relacionan directamente con el contenido del curso. Estos pueden tomar la forma de proyectos grupales o individuales, presentaciones, estudiar a la comunidad, entrevistas sobre temas específicos y una amplia gama de otras actividades. La calidad del trabajo de los estudiantes se juzga de acuerdo con los criterios preestablecidos que se les dan a los estudiantes con anticipación. Estos criterios pueden tomar la forma de rúbricas o pautas específicas a seguir. Estos criterios detallados proporcionan orientación para estudiantes y aumentan su confianza porque saben lo que se espera de ellos (p.443).

Este tipo de técnicas permiten que el estudiante sea participe activo de su proceso de aprendizaje otorgándole oportunidades de poder desarrollar un pensamiento crítico y habilidades efectivas para resolver problemas y la ventaja es que el aprendizaje adquirido es significativo puesto que los estudiantes saben que han realizado un proyecto o actividad que tienden a recordar por mucho más tiempo y además son capaces de transferir el nuevo aprendizaje a situaciones futuras. Por otro lado, es muy útil para el profesor ya que le permite obtener información significativa sobre el conocimiento y las habilidades de los estudiantes y con base en ella actuar en aras de mejorar sus propios procesos de enseñanza (Litchfield y Dempsey, 2013).

Dentro de la evaluación formativa se encuentran 3 ejes importantes que la caracterizan: la evaluación continua, la autoevaluación y la retroalimentación. La 
evaluación continua se desarrolla durante el proceso de aprendizaje a través de diversas actividades evaluativas enfocándose en afianzar aprendizajes, remediar dificultades y prepara al estudiante para usar el lenguaje significativamente en diversos contextos. La autoevaluación es "el proceso mediante el cual el alumno realiza una introspección crítica sobre sus debilidades y fortalezas en el proceso de aprendizaje" (Arias y Maturana, 2005, p.69). El estudiante hace una valoración de conocimientos, destrezas, valores y actitudes. La retroalimentación es la "información que se proporciona a los alumnos sobre si su producción e interpretación del lenguaje es adecuada o no. Esto puede ser en forma de corrección directa, o puede tomar formas más indirectas" (Saville-Troike y Barto, 2016, p.198). La "retroalimentación es probablemente la comunicación más poderosa que los educadores pueden regularmente usar para influir en la competencia del estudiante" (Wlodkowski, 2008, p. 315). Además, implica un dialogo entre docente y estudiante en el cual se proveen comentarios sobre el proceso de aprendizaje del idioma de carácter cognitivo y afectivo con el propósito de reformular los procesos en aras de mejorar la competencia comunicativa en el idioma extranjero por lo que debe caracterizarse por ser precisa, relevante y compleja para que el estudiante sienta que es significativa y orientada hacia sus necesidades. En este proceso, se enfatiza la importancia del conocimiento tácito, conocimiento que se desarrolla en la evaluación formativa, un conocimiento que tanto el profesor como el estudiante obtienen a través de la discusión, la reflexión y la experiencia (Arias y Maturana, 2005; Arias, Maturana y Restrepo, 2012; Voogt y Kasurien, 2005).

Adicionalmente, otro propósito de la retroalimentación formativa es la participación profunda de los estudiantes en estrategias metacognitivas como la planificación de objetivos personales, el monitoreo y la reflexión, para poder supervisar y dirigir el propio aprendizaje con el fin de que el estudiante sea más comprometido, responsable y efectivo. Es decir, influir en el empoderamiento de los estudiantes para llegar a ser estudiantes autorregulados (Clark, 2012).

Gu (2017), considera que para poder poner en práctica la evaluación formativa, el evento evaluativo a diseñar debe contener una serie de elementos a tener en cuenta:

- Propósito de la evaluación: el evento debe ser activado por un propósito intencional y formativo.

- Enfoque de evaluación: el evaluador debe saber exactamente qué se está evaluando y cuáles son los criterios de éxito. En otras palabras, el objetivo de la evaluación debe ser muy claro.

- Método de evaluación: se utiliza una herramienta de evaluación adecuada para obtener evidencia del aprendizaje del estudiante; y tiene que proporcionar suficiente información para informar al asesor.

- Uso de la evaluación: los resultados de la evaluación deberán interpretarse en términos de qué tan lejos está el alumno del objetivo de aprendizaje y cuáles deben ser los próximos pasos para cerrar la brecha. La información se transmite de vuelta al alumno y al profesor como retroalimentación formativa.

- Consecuencia de la evaluación: se deberán proporcionar oportunidades en varias formas (por ejemplo, tareas en el aula, ejercicios adicionales o lecciones de seguimiento) para que los alumnos actúen de acuerdo con la 
retroalimentación a fin de cerrar la brecha entre su estado de aprendizaje actual y el objetivo. (p.17)

\section{Tipos de evaluación formativa}

Gu (2017), indica que hay dos tipos de evaluación formativa: la evaluación formativa planeada y la evaluación formativa interactiva, las dos empiezan con un propósito. En la evaluación formativa planificada se utiliza herramientas de evaluación más formales para lograr que los estudiantes aprendan, seguido de la interpretación del resultado, proporcionando comentarios y tomando acción sobre ellos para cerrar la brecha. Por otro lado, la evaluación formativa interactiva no se planifica, aunque el propósito formativo de obtener evidencia de aprendizaje está en el fondo de la mente del evaluador todo el tiempo. La evaluación formativa interactiva por lo general se lleva a cabo durante el proceso de enseñanza-aprendizaje cuando el maestro identifica problemáticas que afectan el objetivo de aprendizaje y en consecuencia se toman acciones para solucionarlas. Luego, el maestro le brinda al estudiante una respuesta rápida y crea oportunidades sobre el terreno para cerrar la brecha, lo que puede o no ser exitoso. Esto cierra el ciclo de un evento de evaluación formativa, pero a partir de ese momento se recopilan más evidencias, comenzando otro ciclo en ciclos en espiral.

\section{Relación profesor estudiante en la evaluación formativa}

El papel del docente es crucial pues debe prepararse para adaptar sus prácticas pedagógicas a los requerimientos de la evaluación formativa debido a que es una concepción diferente en donde las posturas de evaluación tradicional deben desarraigarse, para dar cabida a una visión más abierta sobre la concepción de evaluación y su proceso. Para lograr este objetivo, los docentes deben asumir una formación continuada en términos de capacitación y actualización de los conocimientos, así como también, el dominio de los procesos que se ven inmersos en la enseñanza aprendizaje "para guiar de manera efectiva, promover la reflexión y promover la practica educativa en cualquier entorno que se le presente, sea en aula regular, aula especial o escenarios no convencionales" (Parra, 2014, p.157).

Asumiendo esta misión, la relación profesor estudiante cambia drásticamente, pues ya no es muy significativa la relación de superioridad del docente con relación al educando (opresor-oprimido), que solía ser un agente receptor pasivo del conocimiento. Al contrario, en los espacios educativos en donde se busque el desarrollo de competencias comunicativas, reflexivas y críticas se debe propender por ofrecer un lugar de mediación para el conocimiento. En este particular Díaz y Hernández (1999), argumentan que el docente debe ser quien organice y sea mediador en el encuentro del estudiante con el conocimiento y su función fundamental es la de orientar y guiar la actividad mental constructiva de sus estudiantes, a quienes brindará asistencia pedagógica ajustada a sus competencias.

En la educación, el aprendizaje de los idiomas, así como la evaluación de ese aprendizaje, se debe construir bajo el dialogo y la reflexión que proporciona el acto crítico. 
Se construye el conocimiento en tanto se comparten saberes entre el maestro y el estudiante en un sentido de igualdad y no de sumisión; en donde el papel del maestro es de guía facilitador quien propicia espacios de autonomía para el aprendizaje. De acuerdo a Philominraj, Ranjan y Diaz (2018),

Ayudar a los estudiantes a ser más autónomos es el papel de un maestro que debe creer que su alumno también es un colaborador en la producción de conocimiento. Esto solo puede suceder si el maestro y los alumnos están en el mismo nivel, donde nadie oprime y nadie está oprimido. Esta propuesta alternativa indica claramente que el estudiante ejerce un control máximo sobre su propio aprendizaje en este sistema descentralizado, y decide no solo qué aprender, sino también cuándo, dónde y cuánto aprender (2018, párr. 32).

La evaluación formativa se lleva a cabo durante el curso y permite recolectar información que servirá de insumo para rediseñar y mejorar los programas de formación de la enseñanza de los idiomas (Richards, 2006) al igual que "sus resultados sirven para que el profesor ofrezca ayuda remedial oportuna o para que cambie el énfasis del curso, si fuera necesario" (Arias y Maturana, 2005, p.69).

\section{Conclusiones}

Tomando como base lo anterior, es importante analizar que, aunque se han dado cambios sustanciales en la manera de enseñar el idioma extranjero todavía se recurre a posturas tradicionales de corte positivista que no logran dar respuesta del proceso completo de aprendizaje a la hora de valorar el progreso de los estudiantes en su desempeño en la lengua extranjera. En consecuencia, se concibe a los estudiantes como simples números en donde se sacan porcentajes de quienes aprueban o no evaluaciones estandarizadas y que dichos resultados son parámetros para juzgar diferentes aspectos del proceso de enseñanza-aprendizaje siendo un tanto injustos con docentes y estudiantes. En consecuencia, la evaluación tradicional no da oportunidades de retroalimentación, que proporcionen verdaderas oportunidades de mejora del proceso de aprendizaje (Brown, 2004; Ketabi y Ketabi, 2014; Çelik y Türkan, 2014). Es decir, el estudiante que es evaluado de forma tradicional y sumativa no tiene la oportunidad de identificar con certeza los aspectos positivos y negativos, así como las debilidades y fortalezas de su proceso de aprendizaje y, por ende, no puede internalizarlo. De igual forma, el docente no puede evidenciar fielmente si sus estrategias y procesos de enseñanza han surtido efecto.

Por otro lado, como se mencionó anteriormente, se aprende los idiomas por motivaciones extrínsecas, en donde no hay una postura internalizada sobre la importancia de aprender una lengua extranjera. Adicional a esto, existe también la frustración que algunos estudiantes experimentan debido a que sus esfuerzos por aprender el idioma no están teniendo los resultados esperados pues las evaluaciones que se les aplican son gramaticales, y abrumantes; y los estudiantes pueden verse afectados por factores afectivos que inciden en su desempeño cognitivo. 
En este sentido, la evaluación formativa ofrece la oportunidad de una valoración mucho más amplia e integral que la sumativa puesto que es constante, ya que se hace un seguimiento a los procesos de enseñanza aprendizaje para evidenciar que está funcionando bien, que no y que problemas deben ser abordados (Richards, 2006). Así como también, es dinámica y práctica, en tanto se pueden tener diferentes instrumentos para evidenciar la competencia que ha logrado el estudiante a lo largo de su proceso educativo; es cíclica, ya que el estudiante puede hacer comparaciones sobre los desempeños obtenidos y los deseados con el propósito de mejorar y, además, permite tomar acciones durante el proceso. Esto implica que la evaluación formativa cumple una función orientadora que permite identificar lo que el estudiante realmente sabe y como lo utiliza en escenarios reales. Este tipo de evaluación toma en cuenta las necesidades de los estudiantes e implica una actividad en la que existe colaboración entre el profesor y el estudiante quienes desempeñan diferentes roles y responsabilidades (Voogt y Kasurien, 2005).

Así, cuando el estudiante se involucra en su proceso de evaluación, surge una internalización del aprendizaje tornándose significativo debido a que el estudiante deja de ser un receptor pasivo del conocimiento y toma acción de su aprendizaje; para esto se auto formula preguntas como: ¿A dónde voy? ¿Dónde estoy ahora? ¿Qué necesito hacer a continuación? (Brookhart y Lazarus, 2017). Se pasa de una educación centrada en el docente a una centrada en el estudiante, en donde aprender el idioma no se limita a repetir frases aisladas del contexto sino a tratar de comunicarse e interpretar el mundo desde la realidad. De igual forma, entra en juego la retroalimentación del profesor hacia el estudiante, así como el monitoreo del estudiante de su propio aprendizaje de idiomas a través de la autoevaluación (Bailey, 2017). Esta actividad influye directamente en el pensamiento del individuo, es decir, en su capacidad para asumir la responsabilidad de su aprendizaje y actuar en consecuencia de manera autónoma para progresar en él y en este proceso lograr transformar la evaluación formativa en autoevaluación. Claro está, que esto se da dependiendo de su participación e inversión de tiempo y esfuerzo. Esto a su vez, conlleva a que el estudiante tome el control y desarrolle experiencia en su propio aprendizaje (Davis, Norris, Malone, y McKay, 2018). Cuando el estudiante logra identificar la brecha entre su estado actual y su meta deseada es cuando está siendo consciente de su aprendizaje, sumado a esto, está también las acciones que tome para cerrar esa brecha y así llegar a su meta deseada (auto regulación); la identificación de esa brecha se activa por lo general después de recibir la retroalimentación (Jamieson, 2011).

Cabe resaltar que, la retroalimentación que se provee al estudiante es muy importante para el aprendizaje. Sin embargo, por sí sola no conduce a una mejora. En este sentido, el cambio se origina si el estudiante decide comprometerse a realizar el ajuste con base en la retroalimentación dada. Ahora bien, este ajuste puede mejorar o no el aprendizaje, pero esto puede cambiar a medida que se va adquiriendo experticia en el proceso. De ahí la importancia de involucrar a los estudiantes en procesos de autoevaluación y autogestión (Gu 2017).

En el desarrollo de la evaluación formativa con los diferentes procesos que ella conlleva, eleva a la evaluación a un nivel más consiente y de autonomía, por ende, el 
estudiante organiza mejor su andamiaje cognitivo, lo que lo lleva a asumir una posición reflexiva y critica sobre su acontecer educativo y en este proceso consiente, aprende también a auto dirigir su aprendizaje a niveles más desafiantes logrando impulsar su autoeficacia (Clark, 2012).

Este proceso, además, logra el desarrollo humanizante de la evaluación en el que tanto el estudiante como el profesor puedan verlo como un espacio de reflexión que logre desarrollar la internalización de los conocimientos, permitiendo identificar las fortalezas para fortalecer la motivación y la autoestima; y las debilidades durante el proceso para ser replanteadas en aras de buscar el mejoramiento del proceso de aprendizaje y por ende un aprendizaje significativo. Asimismo, la evaluación debe ser crítica para brindar retroalimentación que permita avanzar para convertir los conocimientos que se adquieren en algo internalizado, personal que permita la autoformación para la vida (Córdova, 2018). Dichas, así las cosas, la evaluación formativa influye no solo en el desarrollo de la competencia comunicativa, sino que además influye en el desarrollo de la autonomía, la autorregulación y la autoeficacia.

Por otro lado, la evaluación formativa al ser concebida como la evaluación para el aprendizaje logra la movilización del conocimiento más allá de los aspectos gramaticales de los idiomas llevando al estudiante al uso real del idioma, es decir, a ser competente comunicativamente; $y$ que, además, en este proceso se responde a otros factores como son la diversidad y los factores afectivos (motivación y ansiedad) que influyen en el proceso de enseñanza-aprendizaje.

Por consiguiente, dentro de la diversidad están los aspectos culturales, en donde el proceso educativo de un idioma extranjero no puede restringirse a la enseñanza y valoración directa de habilidades lingüísticas solamente. Los modelos contemporáneos de competencia comunicativa muestran que hay mucho más para el aprendizaje de un idioma y que incluye el componente vital del conocimiento y la conciencia cultural de los estudiantes (Nunan 1988). En consecuencia, en la evaluación formativa, los estudiantes en su diversidad muestran lo que tienen aprendido, en lugar de tener que sufrir a través de una evaluación estandarizada que podría ser sesgada culturalmente (Janisch, Liu y Akrofi, 2007).

Igualmente, en referencia a los estilos de aprendizaje la evaluación formativa se ajusta a ellos en tanto el profesor ajusta el repertorio evaluativo para potenciarlos. Asimismo, la evaluación formativa ayuda a identificar los estudiantes que tienen dificultades, y así ajustar la enseñanza de tal forma que se los pueda apoyar (Erling, Ashmore y Kapur, 2016). Además, Brookhart y Lazarus (2017), sugieren que los estudiantes con discapacidades (barreras) pueden beneficiarse de la evaluación formativa gracias a las adaptaciones, y el andamiaje para mejorar su aprendizaje. Similarmente, la evaluación formativa también permite identificar los estudiantes que están llevando un buen proceso para planear como proveerles oportunidades de aprendizaje desafiantes y utilizar diferentes materiales con los cuales puedan progresar.

En cuanto a los factores afectivos, Clark (2012), argumenta que la evaluación formativa logra develar los aspectos internos y por ende "recónditos psicológicos y 
afectivos del proceso de aprendizaje... La combinación de demandas meta cognitivas y contexto social de apoyo explica por qué es un proceso ampliamente reconocido que mejora la motivación y el rendimiento de los estudiantes" (p.213). Por lo anteriormente expuesto, se puede evidenciar que aparte de ser una evaluación formativa se convierte también en evaluación humanizada.

No obstante, uno de los juicios que se le hace a la evaluación formativa es la falta de objetividad. Sin embargo, como lo sustenta Huerta-Macias (2002), aunque los exámenes estandarizados han sido catalogados como objetivos, esa noción ha sido desafiada debido a la procedencia de la mayoría de los exámenes estandarizados que al igual que la evaluación formativa son producidos por humanos y como humanos se tienen prejuicios. Un examen estandarizado representa un acuerdo entre varias personas sobre los procedimientos de calificación, formato y / o contenido para esa prueba específica, es decir, quienes desarrollaron el examen no son realmente objetivos, sino que comparten una misma visión, los mismos prejuicios. Además, los resultados de estas pruebas pueden ser subjetivos porque pueden ser manipulados para reflejar ciertos sesgos de acuerdo a los objetivos de quien la aplica. Por lo tanto, no hay una base sólida para desvirtuar la evaluación formativa.

En lo referente al docente, la evaluación formativa en su esencia humanizante permite al docente recolectar variada y continua información que servirá de sustento para valorar cada esfuerzo que el estudiante hace por utilizar el idioma de una forma constante, y que le permita al mismo tiempo, ser más participe de las mejoras que va teniendo; así como también, de las debilidades que tiene y como hacerles frente. Un proceso de evaluación en donde el estudiante sea reflexivo sobre su proceso educativo. Sin embargo, todavía se necesita transformar el paradigma asociado a la evaluación por parte del profesorado debido a que, aunque se comprende la finalidad de la evaluación formativa; en su aplicación aún se continúa percibiendo el acto de evaluación como un tipo de prueba, de examen, y en consecuencia siguen en la búsqueda del formato correcto de evaluación formativa. "Este es un problema relativamente fácil de resolver, una vez que los maestros se dan cuenta de que la formalidad no reside dentro de la herramienta de evaluación; más bien, cualquier herramienta de evaluación puede usarse de manera formativa" (Gu 2017 p. 22). En otras palabras, una herramienta evaluativa que puede ser sumativa, formal, informal, alternativa o tradicional puede transformarse en una herramienta de evaluación formativa.

En este orden de ideas, es primordial poner en una balanza lo que verdaderamente importa en la enseñanza de los idiomas. Es decir, ¿importa que el estudiante obtenga buenas notas en sus evaluaciones con el objetivo de que apruebe los cursos? ¿O lo que verdaderamente importa es que aprendan el idioma para usarlo a nivel comunicativo efectivamente? Es entonces que las diferentes evaluaciones que se diseñen para valorar el aprendizaje de los idiomas deben brindar a los estudiantes la oportunidad de demostrar su capacidad para utilizar sus recursos lingüísticos para construir e interpretar significados académicos. Es decir, las evaluaciones que se limitan a emitir puntajes y no brindan estas oportunidades de producción significativa, no pueden argumentar que pueden indicar la competencia del estudiante para usar el idioma en 
diversos contextos por lo que no pueden definirse como formativas (Mohan, 2012). De igual manera, el hecho de solo recibir una nota no le permite al estudiante evidenciar y comprender que necesita mejorar (Hattie y Timperley, 2007).

Finalmente, la evaluación formativa implica una verdadera interconexión de los procesos de enseñanza y evaluación permitiendo desarrollar la creatividad del docente, al poner en marcha procesos para diseñar evaluaciones contextualizadas, enfocadas en las necesidades de sus estudiantes, tomando en cuenta aspectos específicos que las evaluaciones estandarizadas por su carácter homogéneo no pueden abordar, logrando de esta forma, desarrollar procesos significativos para potenciar la competencia comunicativa en el idioma extranjero.

\section{Referencias Bibliográficas}

Arias, C. I., Maturana, L. M., \& Restrepo, M. I. (2012). Evaluación de los aprendizajes en lenguas extranjeras: hacia prácticas justas y democráticas. Lenguaje, 40(1), 99126.

Arias, C. y Maturana, L. (2005). Evaluación en lenguas extranjeras: discursos y prácticas. Íkala, revista de lenguaje y cultura, 10(16),63-91. Base de datos Redalyc. https://www.redalyc.org/articulo.oa?id=2550/255020409003

Bailey A.L. (2017) Assessing the Language of Young Learners. En: Shohamy E., Or I., May S. (eds) Language Testing and Assessment. Encyclopedia of Language and Education (3rd ed.). Springer, Cham.

Breen, M. P., \& Candlin, C. N. (1980). The essentials of a communicative curriculum in language teaching. Applied linguistics, 1(2), 89-112.

Brookhart, S., \& Lazarus, S. (2017). Formative assessment for students with disabilities. Commissioned by the Council of Chief State School Officers State Collaboratives on Assessing Special Education Students and Formative Assessment.

Brown, H. D. (2000). Principles of language learning and teaching (Vol. 4). Longman.

Brown, H. D. (2002). English language teaching in the "post-method" era: Toward better diagnosis, treatment, and assessment. Methodology in language teaching: An anthology of current practice, 9-18.

Brown, H. D. (2004). Language assessment: principles and classroom practices. Pearson Education.

Çelik, S., \& Türkan, S. (2014). Assessment and evaluation in EFL: Classroom methods, assessment tools, and the instructional implications of high-stakes exams. En S. Çelik (Ed.), Approaches and principles in English as a foreign language (EFL) education (pp. 419-428). Eğiten.

Cheng, Y. S., Horwitz, E. K., \& Schallert, D. L. (1999). Language anxiety: Differentiating writing and speaking components. Language learning, 49(3), 417-446.

Clark, I. (2012). Formative assessment: Assessment is for self-regulated learning. Educational Psychology Review, 24(2), 205249. https://doi.org/10.1007/s10648-011-9191-6

Coombe, C. (2018). An A to $Z$ of Second Language Assessment: How Language Teachers Understand Assessment Concepts. British Council. https://www.britishcouncil.org/sites/default/files/a to z glossary final.pdf 
Córdova, M. O. (2018). Evaluación de los aprendizajes desde la perspectiva de la teoría crítica. Rev. fac. cienc. méd.(Impr.), 7-9. http://www.bvs.hn/RFCM/pdf/2018/pdf/RFCMVol15-1-2018-2.pdf

Davis, J. M., Norris, J. M., Malone, M. E., McKay, T. H., \& Son, Y.A. (Eds.). (2018). Useful Assessment and Evaluation in Language Education. Georgetown University Press.

Díaz, F., y Hernández, G. (2002). Estrategias docentes para un aprendizaje significativo (Vol. 2). McGraw-Hill.

Ellis, R. (1997). SLA Research and Language Teaching. Oxford University Press.

Erling, E., Ashmore, K., \& Kapur, K. (2016). Supporting Language Learning through Formative Assessment. http://doer.col.org/handle/123456789/5917

Gu, P. (2017). Formative assessment of language learning strategies. 英语学习 (教师版 English Language Learning (Teacher Edition). 2017. 16-24.

Hattie, J. \& Timperley, H. (2007). The power of feedback. Review of Education Research. 77 (1): 81-112.

Herrera-Seda, C. M., Pérez-Salas, C. P., \& Echeita, G. (2016). Teorías implícitas y prácticas de enseñanza que promueven la inclusión educativa en la universidad: Instrumentos y antecedentes para la reflexión y discusión. Formación universitaria, 9(5), 49-64.

Horwitz, E. (2001). Language anxiety and achievement. Annual review of applied linguistics, 21, 112-126.

Horwitz, E. K., Horwitz, M. B., \& Cope, J. (1986). Foreign language classroom anxiety. The Modern Language Journal, 70, 125-132.

Huerta-Macias, A. (2002). Alternative assessment: Responses to commonly asked questions. Methodology in language teaching, 338-343.

Jamieson, J. (2011). Assessment of classroom language learning. Handbook of research in second language teaching and learning, 2, 768-785. Routledge

Janisch, C., Liu, X., \& Akrofi, A. (2007). Implementing alternative assessment: opportunities and obstacles. En The Educational Forum (Vol. 71, No. 3, pp. 221230). Taylor \& Francis Group. Base de datos ERIC. https://files.eric.ed.gov/fulltext/EJ763213.pdf

Katalin, P. (2006). Foreign language classroom anxiety: A classroom perspective. University of Pécs Roundtable 2006: Empirical Studies in English Applied Linguistics, 39.

Ketabi, S., \& Ketabi, S. (2014). Classroom and Formative Assessment in Second/Foreign Language Teaching and Learning. Theory \& Practice in Language Studies, 4(2).

Kormos, J. (2013). Editorial. Assessing L2 students with learning and other disabilities. Cambridge Scholars Publishing. XV.

Larrenua, R. (2015). La motivación en el proceso de enseñanza/aprendizaje de lenguas extranjeras. [Trabajo de fin de grado. Universidad de las Islas Baleares]. http://dspace.

uib.es/xmlui/bitstream/handle/11201/145393/Larrenua_Vegara_Robert o. pdf.

Leiva, J. J. (2013). De la integración a la inclusión: evolución y cambio en la mentalidad del alumnado universitario de educación especial en un contexto universitario español. Actualidades investigativas en educación, 13(3), 605-630. 
Leung C., \& Lewkowicz J. (2017) Assessing Second/Additional Language of Diverse Populations. In: Shohamy E., Or I., May S. (eds) Language Testing and Assessment. Encyclopedia of Language and Education (3rd ed.). Springer, Cham. https://doi.org/10.1007/978-3-319-02261-1 23

Litchfield, B. C., \& Dempsey, J. V. (2013). Alternative Assessment Techniques for Blended and Online Courses. International Association for Development of the Information Society. Base de datos ERIC. https://files.eric.ed.gov/fulltext/ED562371.pdf

Madrid, D. (1997): "La evaluación del área curricular de la lengua extranjera" en H. Salmerón (ed.) (1997): Evaluación Educativa: Teoría, metodología y aplicaciones en áreas de conocimiento, pp. 252-290. Grupo Editorial Universitario. $\mathrm{H}$.

Ministerio de Educación Nacional. (1998). Serie lineamientos curriculares idiomas extranjeros. 89869 archivo pdf4.pdf

Mohan, B. A. (2012). Qualitative research methods in second language assessment. Research methods and methodologies in education, 752-767.

Nunan, D. (1988). The learner-centred curriculum: A study in second language teaching. Cambridge University Press.

Oxford, R. L. (2003). Language learning styles and strategies: An overview. Learning Styles \& Strategies/Oxford, GALA, 2003, 1-25.

Parra, K. (2014). El docente y el uso de la mediación en los procesos de enseñanza y aprendizaje. Revista de Investigación, 38(83),155-180. Base de datos Redalyc. https://www.redalyc.org/articulo.oa?id=3761/376140398009

Pastor, S. (2003). La evaluación del proceso de aprendizaje de segundas lenguas. Perspectivas teóricas y metodológicas: lengua de acogida, educación intercultural y contextos inclusivos. Publisher: Comunidad de Madrid, Consejería de Educación, pp.503-513.

Paz Maldonado, E. J. (2018). La formación del profesorado universitario para la atención a la diversidad en la educación superior. IE Revista de investigación educativa de la REDIECH, 9(16), 67-82.

Philominraj A., Ranjan, R. \& Yañez, D.M. (2018). Paulo Freire y el cambio de paradigma en la enseñanza e el aprendizaje del Idioma: Un estudio de caso sobre ChileIndia. https://doi.org/10.17648/paulofreire-2018-89536

Richards, J. C. (2006). Communicative language teaching today. Cambridge University Press. $\quad$ https://www.professorjackrichards.com/wp-content/uploads/RichardsCommunicative-Language.pdf

Richards, J. C., \& Renandya, W. A. (Eds.). (2002). Methodology in language teaching: An anthology of current practice. Cambridge university press.

Rodríguez, N. (2012). Causas que intervienen en la motivación del alumno en la enseñanza-aprendizaje de idiomas: el pensamiento del profesor. Didáctica. Lengua y literatura, 24, 381-409.

Saville-Troike, M., \& Barto, K. (2016). Introducing second language acquisition. Cambridge University Press.

Shohamy, E. (2017) Critical Language Testing. En: Shohamy E., Or I., May S. (eds) Language Testing and Assessment. Encyclopedia of Language and Education (3rd ed.). Springer, Cham. 
Torelló, Ó. M., \& Olmos, P. (2012). La atención a la diversidad en educación superior: Una perspectiva desde las competencias docentes. Revista de educación inclusiva, 5(1), 159-174.

Voogt, J., \& Kasurien, H. (2005). Finland: Emphasising development instead of competition and comparison. What works in innovation in education. OECD http://www.oecd.org/education/ceri/34260381.pdf

Wlodkowski, R., (2008). Enhancing adult motivation to learn: A comprehensive guide for teaching all adults (3rd ed.). San Francisco: Jossey-Bass. 